\title{
Theoretical Aspects of Vertically Invariant Gray-Level Morphological Operators and Their Application on Adaptive Signal and Image Filtering
}

\author{
Chu-Song Chen, Member, IEEE, Ja-Ling Wu, Senior Member, IEEE, and Yi-Ping Hung, Member, IEEE
}

\begin{abstract}
In this paper, we use vertically invariant morphological filters for time-varying or adaptive signal processing. The morphological filters adopted in this paper are vertically invariant openings and closings. Vertically invariant openings and closings have intuitive geometric interpretations and can provide different filtering scales with respect to different spacial positions. Hence, they are suitable for adaptive signal filtering. To adaptively asssign structuring elements of the vertically invariant openings or closings, we develop the progressive umbra-filling (PUF) procedure. Experimental results have shown that our approach can successfully eliminate noises without oversmoothing the important features of a signal.
\end{abstract}

Index Terms-Adaptive signal processing, mathematical morphology.

\section{INTRODUCTION}

$\mathbf{I}$ $\mathrm{N}$ THE PAST, many approaches have been proposed for edge-preserving signal smoothing. It is well known that because all linear filters confuse and remove the high frequency components of the recovered signals along with the noise, the linear smoothing procedures are no longer a good choice if the signal is subjected to jump changes. For this reason, linear filters cause a typical effect of "edge blurring" when applied to the signals with jumps. Hence, to avoid the effect of edge blurring, nonlinear filters are required. The existing methods for edge-preserving filtering can be classified into the following three categories:

1) Optimization approaches: In an optimization approach, filtering is achieved by minimizing an error function. An error function usually contains two terms: a data term and a smooth term. For example, in [17], an error function was derived based on the statistical analysis with Markov random fields and was then minimized using a GNC algorithm. In [23], two Kalman filters (running forward and backward in time, respectively) coupled in a nonlinear fashion were used to explore a new class of nonlinear edge-preserving filtering algorithms. However, due to the computation burden, an optimization approach is usually difficult to implement efficiently.

Manuscript received June 20, 1996; revised September 1, 1998. The associate editor coordinating the review of this paper and approving it for publication was Prof. Peter C. Doerschuk.

C.-S. Chen and Y.-P. Hung is with the Institute of Information Science, Academia Sinica, Nankang, Taipei, Taiwan, R.O.C.

J.-L. Wu is with the Department of Computer Science and Information Engineering, National Taiwan University, Taipei, Taiwan, R.O.C.

Publisher Item Identifier S 1053-587X(99)02132-7.
2) Rank-order operations: The median filter [8] seems to be the simplest nonlinear edge-preserving smoothing approach and has been generalized to filters that incorporate rank-order operations (multilevel median filters, order statistic filters, stack filters, and so on). The rankorder filter is fast and easy to implement. However, rank-order operations are not appropriate for signal smoothing and noise removal if the signal or noise models are nonstationary.

3) Adaptive smoothing: The concept of adaptive smoothing is to remove noises while preserving features by varying the filtering scales with respect to spatial positions [1], [20], [24], [27], [31]. Hence, an adaptive smoothing method usually contains two major steps: a feature-detection step and a scale-assignment step. In the feature-detection step, the feature parts (e.g., jumping edges) are extracted from a signal; then, in the scaleassignment step, different filtering scales are assigned to the feature parts and the other parts, respectively. The advantage of the adaptive smoothing approach is that it is not only fast but can also be utilized even if the signal or noise models are nonstationary.

In this paper, we develop a new approach for adaptive signal smoothing using vertically invariant morphological filters. In particular, the morphological filters adopted in our work are vertically invariant morphological openings (MV openings) and vertically invariant morphological closings (MV closings), as introduced below. First, we recall some notations and terminologies in mathematical morphology that we will use in the sequel. Let $\Gamma$ be the functional space containing all the functions from $R^{n}$ to $R \cup\{ \pm \infty\}$. By an operator, we mean a mapping $\mathrm{S}: \Gamma \rightarrow \Gamma$. An operator $S$ is said to be

- increasing if $f<g$ implies $S[f]<S[g]$ for all $f, g \in \Gamma$ (where $f<g$ iff $f(x)<g(x)$ for all $x \in R^{n}$ );

- idempotent if $S^{2}=S$ (where $S^{2}=S \circ S$ );

- extensive if $f<S[f]$, and antiextensive if $S[f]<f$.

An operator that is increasing and idempotent is called a morphological filter [29]. An opening (or closing) is a morphological filter that is antiextensive (or extensive).

In this paper, we call an operator $S: \Gamma \rightarrow \Gamma$ vertically invariant if $S[f+a]=S[f]+a$ for all $f \in \Gamma$ and $a \in R$ (where $[f+a](x)=f(x)+a$ for all $x \in R^{n}$ and $a \in R$ ). In other words, an operator is vertically invariant if it is invariant with respect to DC biases. According to [28, Proposition 2.7], 


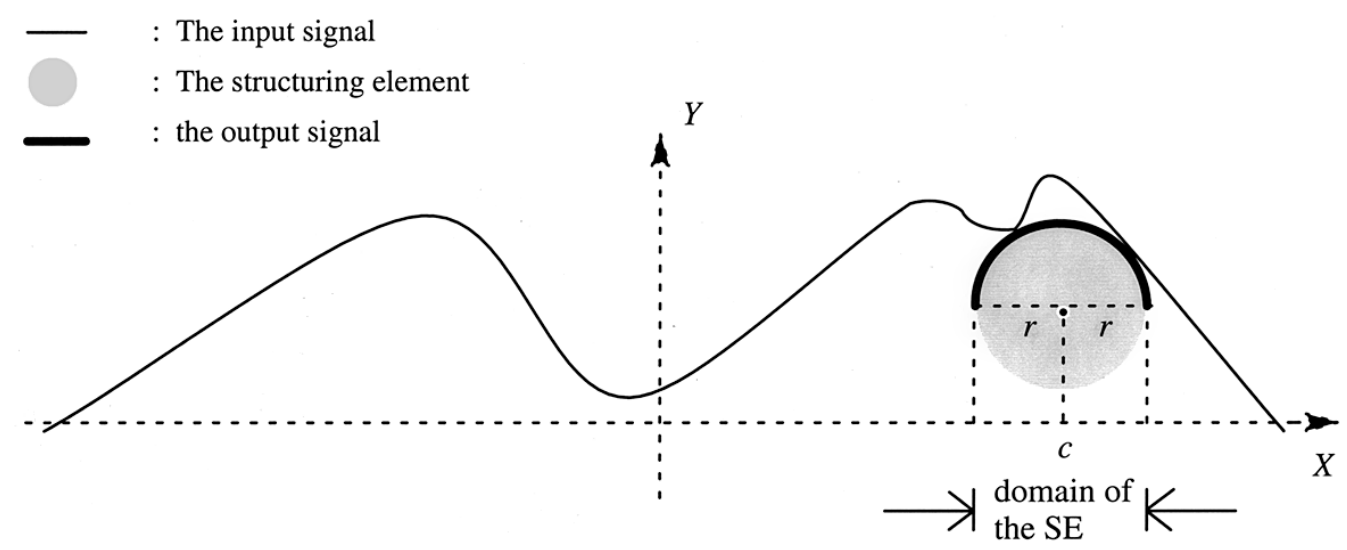

Fig. 1. One-dimensional example of the structural V opening with circular SE.

the following property holds for vertically invariant openings (namely, V openings).

Property 1: Every V opening can be represented as the supremum of a set of structural $V$ openings, where the structural V opening is defined in the following (see [28]).

Definition 1-Structural V Opening: For each function $g \in$ $\Gamma$, the corresponding structural $\mathrm{V}$ opening is defined as

$$
a_{g}^{V}[f]=\operatorname{Sup}\{g+a \mid g+a<f, a \in R\} .
$$

For instance, Fig. 1 shows a 1-D example of a structural $\mathrm{V}$ opening if $g$ is a circular function of radius $r$, that is

$$
g(x)= \begin{cases}\sqrt{r^{2}-(x-c)^{2}}, & \text { if }|x-c| \leq r \\ -\infty, & \text { if }|x-c|>r\end{cases}
$$

In practice, we can also treat a single structural $\mathrm{V}$ opening defined in (1.1) as the $\mathrm{V}$ opening in which $g$ is regarded as a single structuring element (SE).

In this paper, a special kind of $\mathrm{V}$ opening [MV openings (resp. MV closings)] is used for adaptive signal or image filtering. The reason why the MV openings and MV closings are adopted in our work for adaptive signal filtering is owing to their space-varying (or time-varying) nature. MV opening can be computed by the composition of a $\mathrm{V}$ erosion and its adjunctive $\mathrm{V}$ dilation [28] as introduced in the following.

Given a set of SE's $\boldsymbol{k}=\left\{k_{x} \in \Gamma \mid x \in R^{n}\right\}$

Property 2-Computation of $V$ Erosion:

$E_{\boldsymbol{k}}[f](x)=\operatorname{Inf}\left\{f(x+z)-k_{x}(z) \mid z \in R^{n}\right\}, \quad$ for all $f \in \Gamma$.

The $\mathrm{V}$ dilation $D_{k}$, which can cause $\left(E_{\boldsymbol{k}}, D_{\boldsymbol{k}}\right)$ to be an adjunction, is defined as

$$
\begin{aligned}
D_{\boldsymbol{k}}[f](x)=\operatorname{Sup}\left\{f(x-z)+k_{x-z}(z) \mid z\right. & \left.\in R^{n}\right\} \\
& \text { for all } f \in \Gamma .
\end{aligned}
$$

Property 3-Computation of MV Opening:

$$
O_{\boldsymbol{k}}[f]=D_{\boldsymbol{k}}\left[E_{k}[f]\right] .
$$

Property 4-Geometric Interpretation of MV Opening:

$$
O_{\boldsymbol{k}}[f]=\operatorname{Sup}\left\{k_{x}+d<f, d \in R, x \in R^{n}\right\} .
$$

To give a more explicit explanation, Fig. 2(a) shows a 1-D example of MV openings using a set of radius-varying circular
SE's. That is, $O_{\boldsymbol{k}}[f]$ can be visualized as sliding $k_{x}$ under $f$, with the shape of $k_{x}$ varying by position $x$, and where the locus of all the highest points reached by some part of $k_{x}$ ( $x \in R^{n}$ ) during the slide then constitutes the MV opening result. MV closing is a dual operator of MV opening, and its geometric interpretation is shown in Fig. 2(b).

MV openings (or MV closings) are specified morphological filters that have great potential for signal filtering applications. In particular, they can be appropriately used for adaptive signal smoothing. This comes mainly from their following three characteristics.

1) They are invariant with respect to DC biases.

2) Their filtering scales can be varying with spatial axes (or time axis in the 1-D case) by using different shapes or sizes of SE's in different spatial positions.

3) Their behavior can be explained by easy-to-understand geometric interpretations (as described in Property 4).

In principle, according to Properties 3 and 4, using MV openings (or MV closings) for signal processing applications is equivalent to finding a set of SE's $\boldsymbol{k}$ with respect to different spatial positions. This is called the SE assignment in this paper. Solution of the SE-assignment problem is a critical issue if MV openings (or MV closings) are used for adaptive signal processing.

In practice, signals usually cannot be modeled in a stationary manner; therefore, a good filtering scheme should be able to adapt to the local data characteristics. Remember that the definition of adaptive smoothing is that of making the filtering scales adaptive to the local property of each position of a signal. A good adaptive-smoothing method should be able to eliminate noises without oversmoothing the important features of signals. To achieve this goal, an useful scale-assignment scheme in adaptive smoothing is to smooth the highly varying parts (or feature parts) with smaller filtering scales and to smooth the flat parts with larger scales [5], [12], [20], [25]. In fact, this is equivalent to assigning smaller SE's to the highly varying parts and larger SE's to the flat parts if an MV opening (or MV closing) is used. In this paper, we develop an adaptive signal smoothing approach based on MV openings and MV closings. We propose the progressive umbra-filling (PUF) procedure to solve the SE-assignment problem. The PUF procedure can be intuitively realized to reconstruct the 


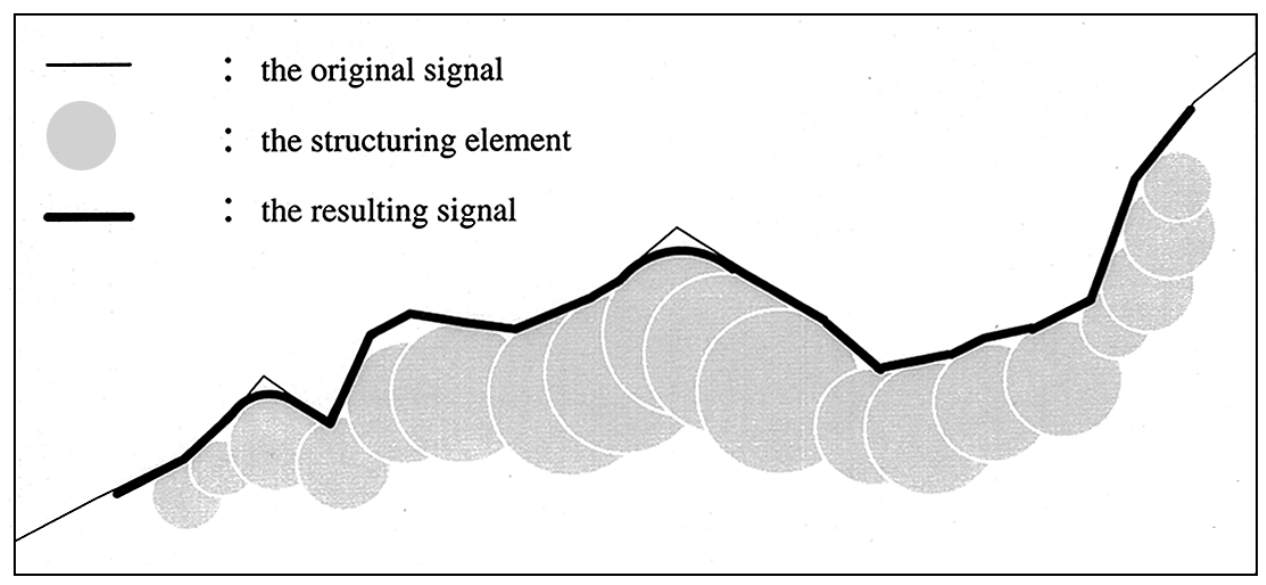

(a)

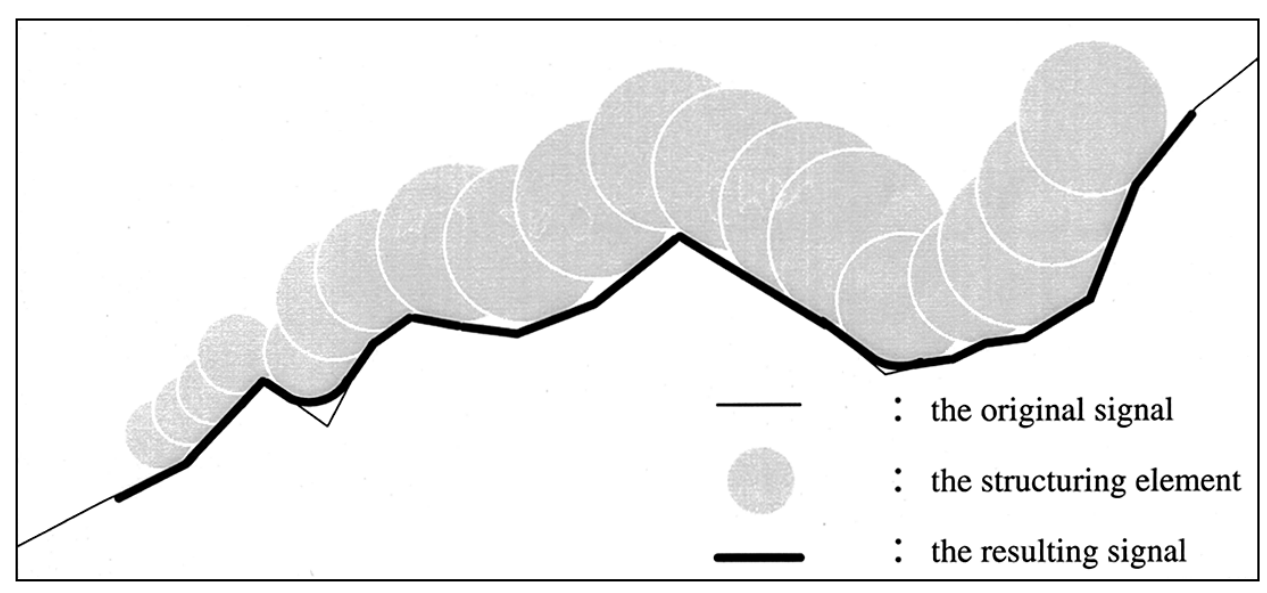

(b)

Fig. 2. (a) Geometric interpretation of MV opening using radius-varying circles. (b) Geometric interpretation of MV closing using radius-varying circles.

umbra of a signal using many size-varying SE's. During the reconstruction process, a coarse-to-fine representation of the signal can be obtained as well. In each level of the coarse-tofine representation, a connected morphological operator [14] is used to extract features and remove irrelevant noises ${ }^{1}$.

This paper is organized as follows. In Section II, the PUF procedure is introduced, which can be effectively used for adaptive signal and image filtering. In Section III, experimental results are presented. Finally, conclusions and discussions are presented in Section IV.

\section{MV OPENINGS AND MV CLOSINGS IN ADAPTIVE SignAL FILTERING}

As mentioned above, a critical issue of applying MV openings (or MV closings) for signal processing is the SE

\footnotetext{
${ }^{1}$ In our early work, MV openings and MV closings are referred to as spacevarying openings and space-varying closings [2], [6]. In [2], we have proposed an adaptive filtering method for smoothing noisy range images using a single MV opening (or MV closing). In [6], we have also performed a simplified statistical analysis of MV openings using flat SE's by adopting a standard analysis method proposed in [21] and [22]. However, in our experience, it is easily affected by noise if only a single MV opening is used for adaptive signal filtering because the SE assignment is usually not an easy task in this case. Hence, in this paper, we use multiple MV openings (or MV closings) in a hierarchical process, which can solve the SE-assignment problem in a more stable way.
}

assignment. Since it is desirable to remove noise while preserving the original signal shape as much as possible, a reasonable guideline for selecting the SE's in MV openings (or MV closings) is that small-size SE's should be assigned to positions containing important features (such as the highfrequency or highly varying parts of a signal); on the other hand, all SE's assigned to positions with low frequency (such as low-variation parts or flat regions) should be large enough because small noises may cause large SNR degradation in flat regions. To achieve this goal, a quantitative measure is required to estimate the variation in each position. In fact, to independently treat the variation estimation phase and the filtering phase as two separate processes could be inefficient. Hence, in this paper, we unify these two phases into a wellstructured procedure, i.e., the PUF procedure. Without lost of generality, we describe the PUF procedure only with MV openings in the following. Notice that the PUF procedure can also be easily modified to use MV openings.

\section{A. The PUF Procedure}

Before introducing the PUF procedure in detail, let us look at a simplified example. In Fig. 3(a), the umbra of a given signal is filled with many overlapped circular SE's of the same scale. Those SE's are treated here as basic units to reconstruct 


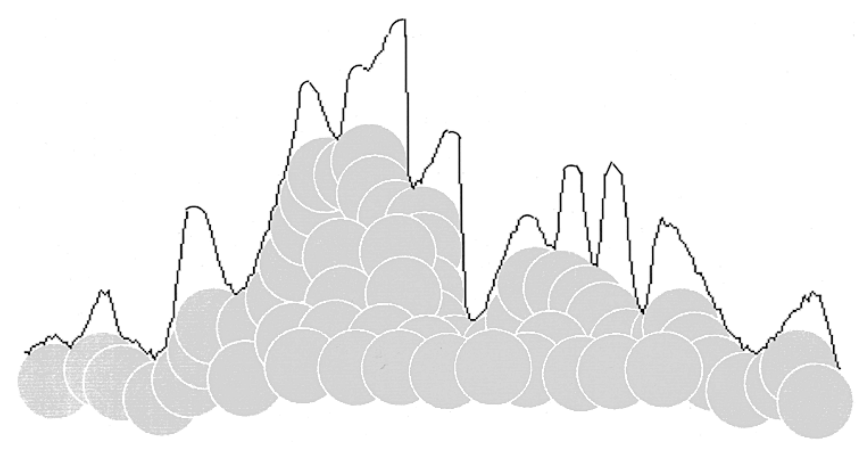

(a)

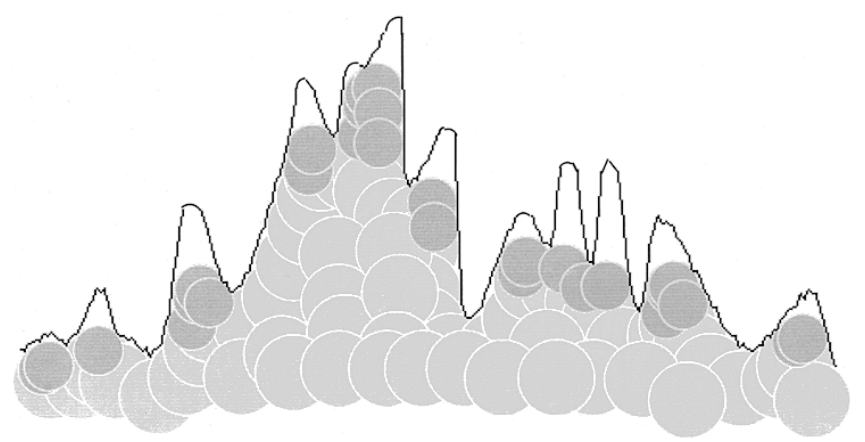

(b)

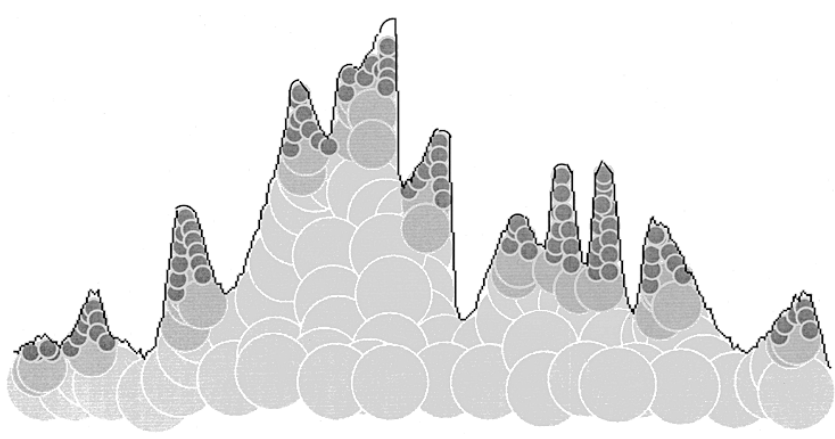

(c)

Fig. 3. Interpretation of the PUF procedure (a special case that the same size of SE's are used with each spatial position in each iteration): (a) Filtering with the largest scale SE's. (b) Reconstruct the feature regions of (a) using middle scale SE's. (c) Reconstruct the feature regions of (b) using the smallest SE's.

the umbra. Since the SE's used in Fig. 3(a) are large in size, small bumping noises can be considerably deleted, whereas some highly varying regions that cannot be filled in with SE's of this scale will remain unchanged. In Fig. 3(b), some of the regions remaining in Fig. 3(a) (which are considered to be feature regions) are filled with SE's of smaller sizes. Finally, in Fig. 3(c), some fine features are constructed by the smallest SE's; hence, the filtered result is verly similar to the natural shape of the given signal. The total effect of the above hierarchical procedure is that it tends to reconstruct the shape of the given signal. In fact, any shape can be reconstructed with such a procedure if the smallest size of the SE degenerates to a single point. Using this filtering process, important features can be preserved, and irrelative noises are removed. Although a morphological opening is used in the above description, this method can be easily extended to the use of morphological closing.
The PUF procedure is an iterative process. In each iteration, a specified MV opening is used to filter the signal obtained from the last iteration. According to the geometric interpretation of MV openings, an MV opening can be completely described by specifying the SE used in each position. In the implementation of the PUF procedure, the class of SE's used is $\{r k \mid r \in R, 0 \leq r \leq \boldsymbol{r}\}$, where

$k \in \Gamma$ convex function;

$r \quad$ size of the $\mathrm{SE}$;

$\boldsymbol{r} \quad$ largest size allowed to be used (which is selected depending on applications).

Hence, the MV openings used in the PUF procedure can be specified by a function $s: R^{n} \rightarrow[0, \boldsymbol{r}]$, where $k_{x}=s(x) k$ is the SE assigned to position $x \in R^{n}$. Here, we call function $s(\cdot)$ the scale-function of this MV opening, and $k$ is referred to as the mother SE, respectively. In the PUF procedure, the domain of the selected mother SE (that is, $\left\{x \in R^{n} \mid k(x)>-\infty\right\}$ ) should be bounded, and $l(k)$ is used to denote the area of the domain of the mother SE $k$. For example, in Fig. $1, l(k)$ is equal to $2 r$. Assume that $f$ is the input signal. Some operations to be used in the PUF procedure is formally defined as follows.

- $O_{s}$ (a mapping from $\Gamma$ to $\Gamma$ ): the MV opening whose scale function is $s(\cdot)$;

- $e_{s}$ (a mapping from $\Gamma$ to $\Gamma$ ): $e_{s}[f]=f-O_{s}[f]$, which is the opening residue of $f(f \in \Gamma)$ with respect to the scale function $s$;

- $D$ (a mapping from $\Gamma$ to $\Gamma_{b}$ ), where $\Gamma_{b}$ is the functional space containing all of the functions from $R^{n}$ to $\{$ True, False $\}): D[f]$ is the extraction of the feature parts of a function $f(f \in \Gamma)$, where $D[f](x)=$ True implies that $x$ is a feature point; otherwise, $x$ is not a feature point. The extraction procedure used in our work is based on a morphological connected-operator, which will be described in detail in Section II-B;

- $F$ (a mapping from $\Gamma$ to $\Gamma$ ): $F[f]$ is the featurization of $f(f \in \Gamma)$ that

$$
F[f](x)= \begin{cases}f(x), & \text { if } D[f](x)=\text { True } \\ 0, & \text { if } D[f](x)=\text { False }\end{cases}
$$

- $A$ (mapping from $\Gamma$ to $R$ ): $A[f]$ is the scale function assigned for the MV opening used for filtering. The principle used for scale-assignment is described in detail in Section II-C.

The basic hierarchy of the PUF procedure can be described as follows.

\section{Basic hierarchy of the PUF procedure}

1) Input a signal $f$.

2) Initially, let the scale function $s(x)=\boldsymbol{r}$ for all $x \in R^{n}$ (i.e., the largest scale is assigned to each position in the beginning). In addition, let the intermediate signal $h=f$, and let the iteration counter $i=0$.

3) While $(s(x)$ is not a zero function), do 2.1-2.8

a) [MV opening using the assigned scales] $g=O_{s}[h]$.

b) [compute the opening residue] $e=e_{s}[h]=h-g$.

c) [feature-parts extraction and featurization of $e$ ] compute $e^{\prime}=F(e)$.

d) [restoration of the feature parts] $h^{\prime}=g+e^{\prime}$. 


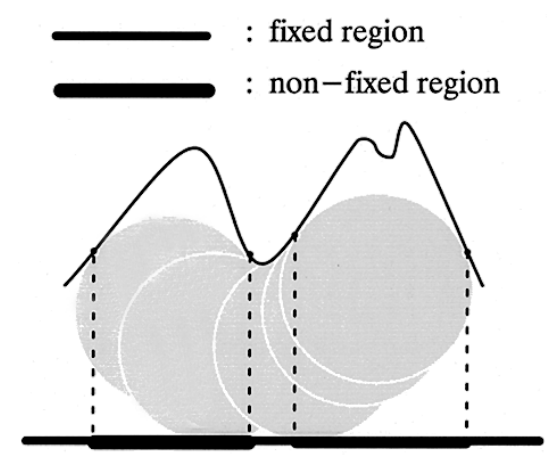

(a)

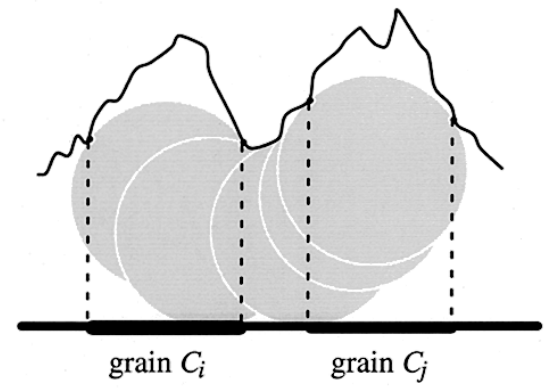

(b)

Fig. 4. Interpretations of the fixed region, the nonfixed region, and the grain.

e) $h \leftarrow h^{\prime}$

f) [scale reassignment] Reassign the SE's of the MV opening to be used in the next iteration according to

$$
s^{\prime}(x)= \begin{cases}k_{i} \cdot A\left[e^{\prime}\right](x) \cdot \gamma, & \text { if } D[f](x)=\text { True } \\ 0, & \text { otherwise }\end{cases}
$$

for all $x \in R^{n}$, where $k_{i}$ is a constant, $0<k_{i} \leq 1^{2}$, and $k_{i+1} \leq k_{i}$ for all $i$.

g) $s \leftarrow s^{\prime}$.

h) $i \leftarrow i+1$.

4) Output $h$.

5) End. ${ }^{2}$

There are two major steps in the PUF procedure. One is the featurization of the opening residue, i.e., the computation of $F[e]$, and the other is the reassignment of the scale function $A\left[e^{\prime}\right]$. They will be described in detail in Sections II-B and II-C, respectively.

\section{B. Extraction of the Feature Parts}

For each $x \in R^{n}$, if $e(x)=0$, then $x$ is labeled as a fixed point; else $x$ is a nonfixed point. Let the fixed region be the set consisting of all the fixed-points, and let the nonfixed region be the set consisting of all the nonfixed points, as shown in Fig. 4(a). In principle, the signal within a nonfixed region can be referred to as the convex highly varying parts of a signal. Assume that the nonfixed region can be divided into $n_{i}$ connected subregions, namely, $C_{1}, C_{2}$, cdots, $C_{n i}$, where $C_{j} ; \cap C_{k}=\Phi$ for all $j, k \in\left\{1,2, \cdots, n_{i}\right\}$. Each $C_{j}$ is referred to as a grain, and each segment of the intermediate signal $h_{j ; i} \in \Gamma$

$$
h_{j ; i}(x)= \begin{cases}h(x), & \text { if } x \in C_{i} \\ -\infty, & \text { otherwise }\end{cases}
$$

is referred to as a connected highly varying portion, as shown in Fig. 4(b). Basically, there is a unique partition $C_{1}, C_{2}, \cdots, C_{n i}$ that satisfies that each $C_{i}$ is connected and that each pair $\left(C_{i}, C_{j}\right)$ is disjoint. A connected operator [14] is an operator that coarsens this partition for every input signal. In particular, the connected operators used in our work are grain operators, where an operator is called a grain operator if it has the following "local property": The value of the output

\footnotetext{
${ }^{2}$ Basically, $k_{i}$ is a damping factor depending on the iteration counter $i$.
}

signal at a given point $x$ is exclusively determined by the zone of the partition of the input signal that contains $x$ [14], where a zone of a set $A$ is a connected component of either $A$ or $A^{c}$.

Grain operators are used for the extraction of the feature parts. For each region $C_{m} m \in\left\{1,2, \cdots, n_{i}\right\}$, a measure $B(\cdot)$ is used to evaluate its fitness of being a feature. If $B\left(C_{m}\right)$ is larger than a given threshold $T_{i ; m}$, then feature $\left(C_{m}\right)=$ True; else feature $\left(C_{m}\right)=$ False. Basically, $T_{i ; m}$ is allowed to be varying with the iteration counter $i$. Some possible selections of $h\left(C_{m}\right)$ are discussed below. For example, we have the following:

volume of $C_{m}$

$$
B\left(C_{m}\right)=\text { volume }\left(C_{m}\right)=\sum_{x \in C_{m}} e(x)
$$

energy of $C_{m}$

$$
B\left(C_{m}\right)=\operatorname{energy}\left(C_{m}\right)=\sum_{x \in C_{m}} e^{2}(x)
$$

height of $C_{m}$

$$
B\left(C_{m}\right)=\text { height }\left(C_{m}\right)=\max \left\{e(x) \mid x \in C_{m}\right\}
$$

or

area of $C_{m}$

$$
B\left(C_{m}\right)=\operatorname{area}\left(C_{m}\right)=\sum_{x \in C_{m}} 1 .
$$

The following equation is used to extract the feature parts:

$$
D[h](x)= \begin{cases}\text { True, }, & \text { if there is an } m \in\left\{1,2, \cdots, n_{i}\right\} \\ & \text { such that } x \in C_{m} \\ & \text { and feature }\left(C_{m}\right)=\text { True } \\ \text { False, }, & \text { otherwise. }\end{cases}
$$

After that, $F[h]$ can then be computed by (2.1).

\section{Scale Reassignment}

In the above, the current signal was filtered using an MV opening and then was restored by adding back the feature part of it. In this step, a new scale function will be assigned for filtering the new generated signal in the next iteration. Since each of the restored connected highly varying portion is smaller than the SE used to filter them in the current iteration, smaller scales have to be assigned for filtering the restored 


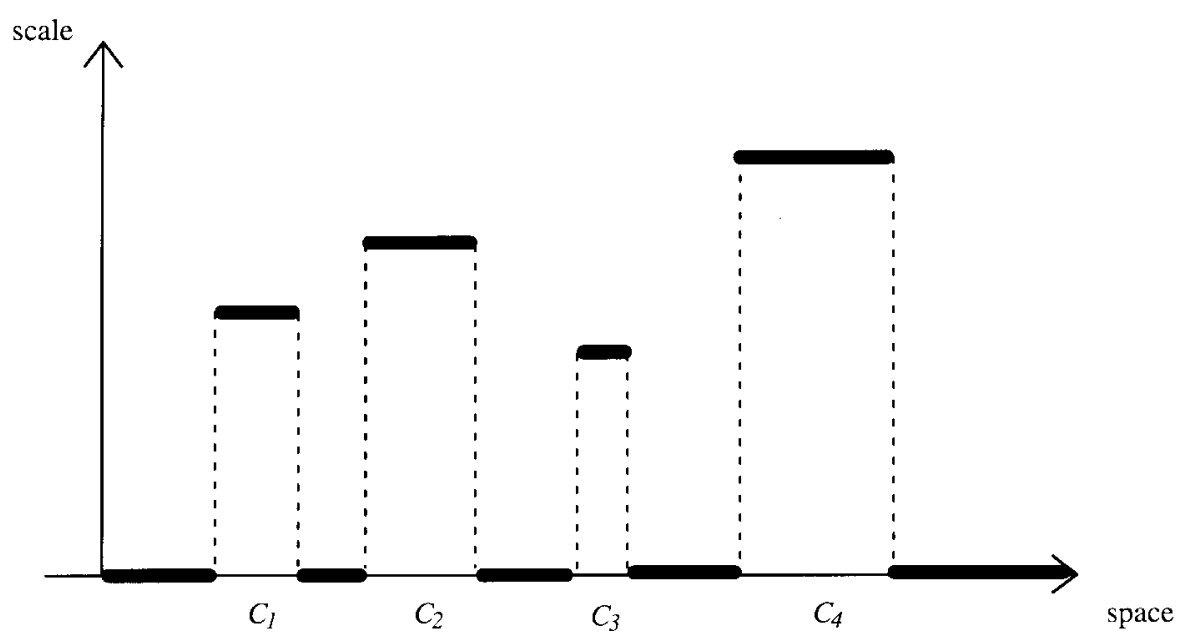

Fig. 5. One-dimensional example of the scale function assigned in an iteration of the PUF procedure. The scale function is piecewise constant. Each grain $C_{j}$ (here, four grains are shown) corresponds to a nonzero piece. The larger the widths of the grains, the larger the scales assigned.

parts in the next iteration. The scale reassignment function used in the PUF procedure is

$$
\begin{aligned}
& A\left[e^{\prime}\right](x) \\
& \quad= \begin{cases}k_{a} \cdot\left(\text { area }\left(C_{m}\right) / l(k)\right), & \text { if } D[f](x)=\text { True and } \\
0, & x \in C_{m}\end{cases} \\
& \text { if } D[f](x)=\text { False }
\end{aligned}
$$

where $k_{a}$ is a constant, $0<k_{a} \leq l(k) /$ area $\left(C_{m}\right)$

Remember that $l(k)$ is the area of the domain of the function $k$. Basically, area $\left(C_{m}\right) / l(k)$ is the ratio of the area of the grain $C_{m}$ to $l(k)$. Hence, by using a proportional constant $k_{a}<1$, it follows that the smaller SE's will be assigned to the narrower connected highly varying portions and vice versa. In particular, the illustrative example shown in Fig. 3 is a special case that $k_{a}=l(k) / \operatorname{area}\left(C_{m}\right)$ (but $k_{i}<1$ ), i.e., the scale reassignment only depends on the iteration counter. In this special case, the scales assigned to the feature regions (i.e., $\left\{x \in C_{m} \mid m=1,2, \cdots, n_{i}\right\}$ ) in each iteration are all the same. However, in general, the scales assigned for the feature regions in each iteration are allowed to be different in the PUF procedure.

It is obvious that the PUF procedure introduced below can be easily modified to use MV closings. In the following, PUF opening is used to denote the PUF procedure using MV opening, and PUF closing is used to denote the PUF procedure using MV closing, respectively. In practice, we usually adopt the combined procedure PUF close-open (PUF closing after PUF opening) or PUF open-close (PUF opening after PUF closing) for signal filtering in real applications.

\section{Piecewise-Constant Scale Function}

Notice that by using (2.6), the scale function of the MV opening used in each iteration is a piecewise constant function. That is, the same size SE's are used to filter the same connected highly varying portions. In principle, in the $i$ th iteration, the newly assigned scale function has $n_{i}$ nonzero pieces, whereas others have zero pieces (as shown in Fig. 5). Because the outputs of the MV opening are the same as the input signals in the region of the zero pieces, only the outputs of the regions of the nonzero pieces have to be computed. Hence, the computation can be speeded up because only the nonzero pieces really have to be considered in implementation.

In fact, if the scales assigned in a piece are all the same with spatial positions, an MV opening degenerates to as a shift-invariant opening. A shift-invariant opening is an MV opening whose SE's are the same with respect to every spatial positions. In fact, shift-invariant openings are widely used in image and signal processing [7], [16], [18], [19], [30] and are introduced by many textbooks [10], [11], [13]. ${ }^{3}$ Hence, the PUF procedure can also be equivalently implemented using shift-invariant openings by considering that the scale function (of the MV opening) assigned in each iteration is piecewise constant.

\section{E. Example}

A 1-D signal is used to examine the effectiveness of the PUF procedure. This signal consists of part of a triangular wave and part of a square wave, which can be referred to as a roof edge and a step edge in an image, respectively. A random noise, which is uniformly distributed in $[-5,5]$ is added to this signal, as shown in Fig. 6(a). The class of SE's used in this experiment is the circular one, that is $\{r k \mid r \in R\}$, where the mother SE $k$ is selected to be a unit circular-function as defined in

$$
k(x)= \begin{cases}\sqrt{1-x^{2}}, & \text { if }|x| \leq 1 \\ -\infty, & \text { if }|x|>1\end{cases}
$$

Both the height and the area of a connected highly varying portion [as, respectively, defined in (2.4) and (2.5)] are used

\footnotetext{
${ }^{3}$ In fact, the PUF procedure can also be described via shift-invariant openings. However, since the concept of the spatially variant scale assignment can be more concisely explained using MV openings rather than using shiftinvariant ones, the MV openings are used to explain the PUF procedure in this paper. In addition, the use of MV openings has the advantage that it has the potential to be generalized to the case that the scale functions assigned in each iteration are not piecewise constant.
} 

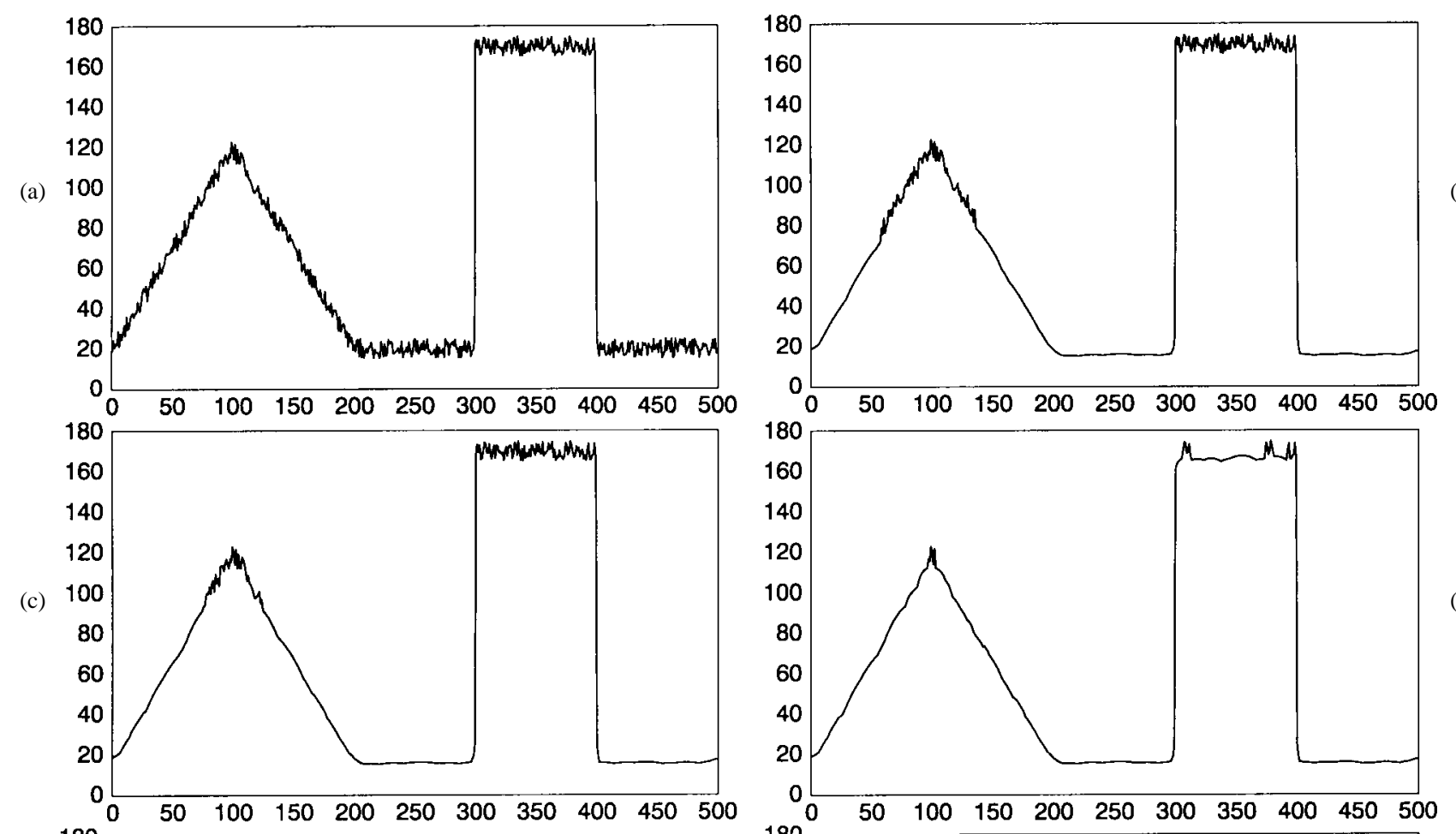

(b)
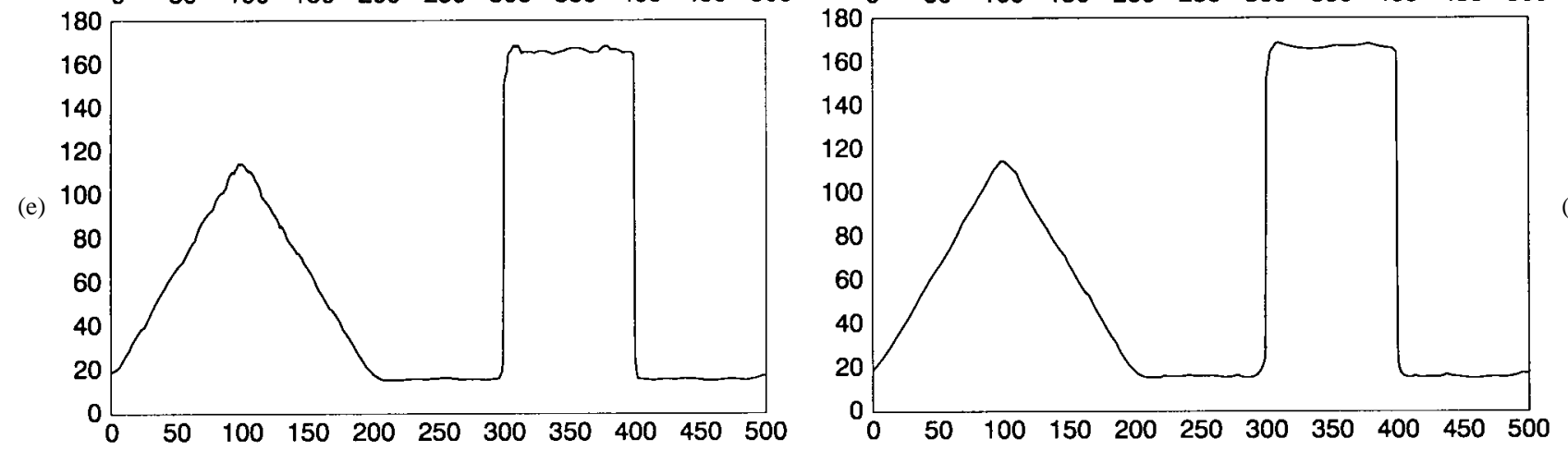

Fig. 6. (a) Signal contains a roof edge and a step edge, which is corrupted by a random noise distributed in [-5, 5]. (b) Filtering result after the first iteration using PUF opening. (c) Filtering result after two iterations of using PUF opening. (d) Filtering result after five iterations using PUF opening. (e) Final filtering result after eight iterations of using PUF opening. (f) Output filtering result after using PUF close-open.

for feature detection, as shown in

$$
B\left(C_{m}\right)= \begin{cases}1, & \text { if height }\left(C_{m}\right)>T_{i ; m}^{H} \text { and } \\ & \text { area }\left(C_{m}\right)>T_{i ; m}^{A} \\ 0, & \text { otherwise }\end{cases}
$$

where $T_{i ; m}^{H}$ and $T_{i ; m}^{A}$ are referred to as height threshold and size threshold, respectively. In our settings, these thresholds (i.e., $T_{i ; m}^{H}$ and $T_{i ; m}^{A}$ ) are also varied with the iteration number $i$. In each iteration of the PUF procedure, $T_{i ; m}^{H}$ and $T_{i ; m}^{A}$ are reduced according to half of the current SE size until they reach some predefined minimal values, as shown in

$$
\begin{aligned}
& T_{i ; m}^{H}=\min \left[0.5 \cdot s(x) \cdot l(k), a_{s}\right], \quad x \in C_{m} \\
& T_{i ; m}^{A}=\min \left[0.5 \cdot s(x) \cdot l(k), a_{h}\right], \quad x \in C_{m}
\end{aligned}
$$

where $a_{s}$ and $a_{h}$ are the predefined minimal values. The advantage of varying these thresholds adaptively with respect to the current SE size is that the noises occurred in large slow-varying regions can be better removed because larger thresholds are applied to them; likewise, the small features occurring in the highly varying region can be better preserved by applying smaller thresholds. The largest radius used in this experiment is $\boldsymbol{r}=100$, and the SE size-reduction factors used are $k_{a}=0.5$ and $k_{i}=1$. The experimental results are shown in Fig. 6(b)-(f). In Fig. 6(b)-(d), the filtering results using the PUF opening after iterations 1,2 , and 5 are shown. In these consecutive results, noises were gradually reduced, and the important features in this signal were preserved precisely. The PUF opening procedure converged at the eighth iteration in this experiment, and the converged result is shown in Fig. 6(e). Finally, the result of using PUF close-open is shown in Fig. 6(f). Generally, this experiment shows that the PUF procedure can remove noises in a progressive way and, hence, can be effectively used for adaptive signal filtering and noise removal. 


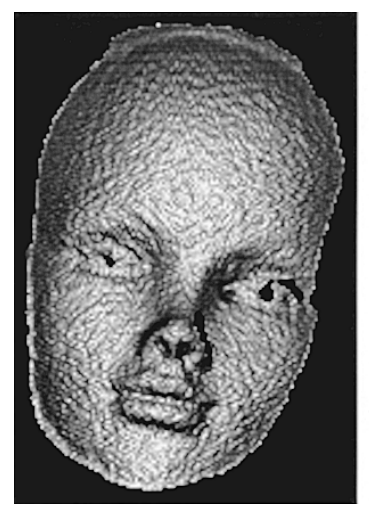

(a)

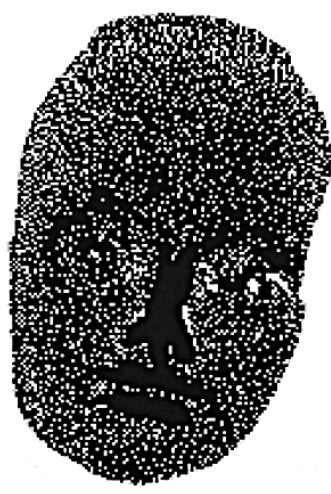

(b)

Fig. 7. (a) Original range image of a model head. The height of this model head is about $200 \mathrm{~mm}$. (b) Nonfixed region (shown in black) of (a) obtained by using the 2-D PUF procedure using MV openings after the first iteration with a spherical SE with radius $10 \mathrm{~mm}$.

\section{F. Computational Complexity}

Assume that in the first iteration of the PUF procedure, the signal to be filtered is quantized to $l_{n}$ data points, and the average length of the SE's used in the MV opening contains $l_{m}$ data points. Basically, the computational complexity of an MV opening is $O\left(l_{m} \cdot l_{n}\right)$. Suppose that in the $i$ th iteration of the PUF procedure, there are $l_{n ; i}$ data points in total contained in the nonzero pieces, and the average size of the SE used can be quantized to $l_{m ; i}$ data points. Then, the computational complexity in the $i$ th iteration is $O\left(l_{m ; i} \cdot l_{n ; i}\right)$. Hence, the total complexity of the PUF procedure is $t_{\mathrm{PUF}}=\sum_{i=1}^{t} O\left(l_{m ; i} \cdot l_{n ; i}\right)$, where $t$ is the number of iterations. Driving the exact form of the computational complexity is not an easy task because the terms $t, l_{m ; i}$ and $l_{n ; i}$ are all signal dependent. In principle, $l_{m ; i}$ and $l_{n ; i}$ will decay rapidly with respect to the iteration number $i$ for most cases. If the decay of $l_{m ; i}$ and $l_{n ; i}$ can be approximately modeled by an average fraction factor $d(d<1)$, i.e., $l_{m ; i}=d \cdot l_{m ; i-1}$ and $l_{n ; i}=d \cdot l_{n ; i-1}$, and assume that $t \rightarrow \infty$, then $t_{\mathrm{PUF}}=(1 / 1-d) \cdot O\left(l_{m} \cdot l_{n}\right)$. For example, if $d=0.5$, then $t_{\text {PUF }}=2 \cdot O\left(l_{m} \cdot l_{n}\right)$. Since $1 / 1-d$ is a constant, the computational complexity of the PUF procedure is roughly $O\left(l_{m} \cdot l_{n}\right)$, which is equal to that of a single MV opening. Consequently, although the PUF procedure uses multiple MV openings iteratively, its computational complexity is still the same as that of a single MV opening in the case that the total area of the domains to be processed in each iteration is exponentially decayed.

\section{G. Residue-Analysis Strategy}

In fact, the PUF procedure can be viewed as a method that is based on the residue-analysis strategy. Usually, a residueanalysis strategy contains three main steps in one iteration:

i) computing the opening-residues or closing-residues;

ii) identifying each local portion of a signal as a feature or noise based on these residues;

iii) restoring features from the opening (or closing) results.

Hence, an inherent assumpption in a residue-analysis strategy is that features and noises can be separated by using openings (or closings) with SE's of different sizes. In the past, residue analysis strategies have also been adopted in some research
[24], [32]. In [32], an iterative-decomposition approach was proposed for texture classification and segmentation. The advantage of using a residue-analysis strategy is that small components with high contrast will be preserved in the segmentation result as well. In [24], an algorithm for noise reduction of intensity images based on a residue-analysis approach has also been proposed. A significant difference between their approaches and the PUF procedure is that the previously cited methods use the shift-invariant opening (or closing) in each iteration; therefore, SE's of the same size are used for each position in the same iteration (somewhat like the case shown in Fig. 3). On the other hand, the PUF procedure uses an MV opening (or MV closing) in each iteration; therefore, the SE size can be adaptively varied with respect to the size of each connected highly varying portion.

\section{H. Dimensionality Considerations}

If the dimenstion of the SE's used in a PUF procedure is $N$, then it is called an $N$-dimensional ( $N$-D) PUF procedure. An important property is that in each iteration of the 1-D PUF procedure, the area of each grain [defined as (2.5)] is smaller than the area of the domain of the used SE. On the other hand, in the $N$-D cases $(N \geq 2)$, the area of each grain may be larger than that of the domain of the SE. For example, Fig. 7 shows the nonfixed region of a range image obtained by using the 2-D PUF procedure (after the first iteration). In Fig. 7(b), almost all the points in the nonfixed region are connected to each other and, hence, are contained in the same grain. The area of this grain is, then, very large, and both noises and features are possibly contained in it. In this case, noises contained in this grain (in particular, slender noise stripes) will be preserved in the current iteration. Hence, in the 2-D PUF procedure, some unremovable noises may remain in each iteration, which will significantly reduce the quality of the obtained filtering result.

Therefore, in this paper, we suggest using 1-D PUF procedure for signal filtering. In fact, to filter a 2-D signal, using 2-D window operators is not definitely necessary. In our approach, the row-column decomposition of this signal is performed, and each row (or column) signal is filtered using a 1-D PUF procedure. It is worth noting that filtering 2-D signals using row-column decompositions has been widely adopted in 


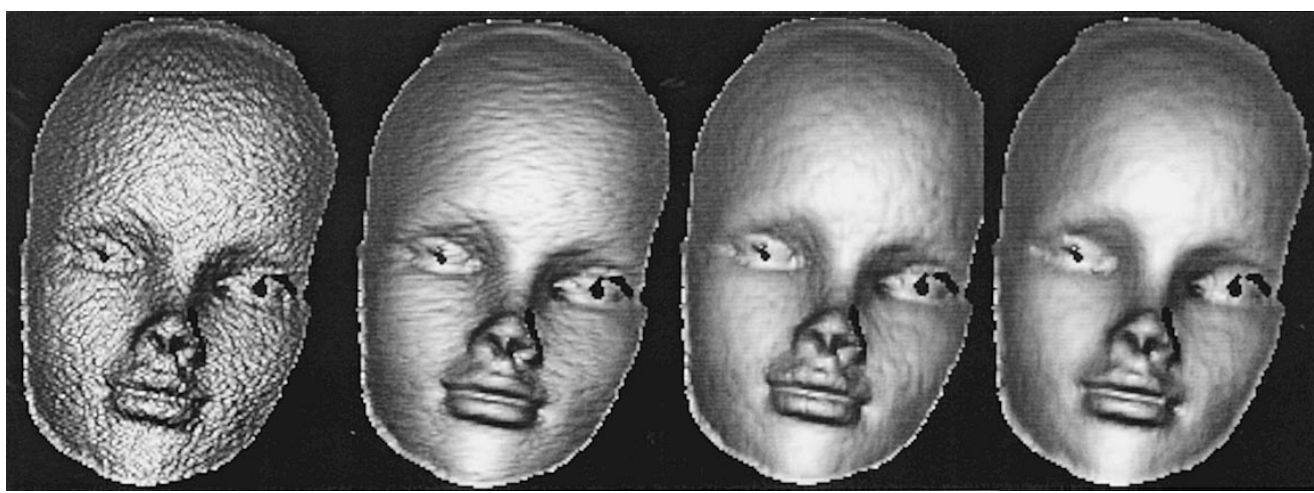

(a)

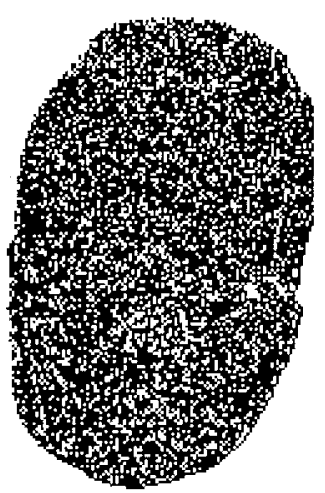

(e)

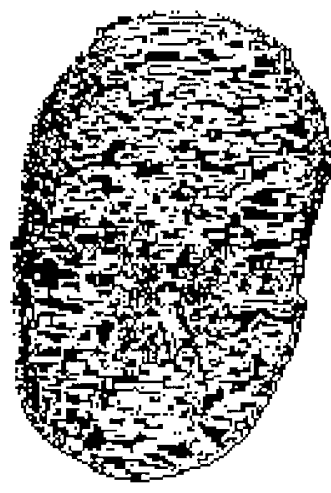

(f) (c)

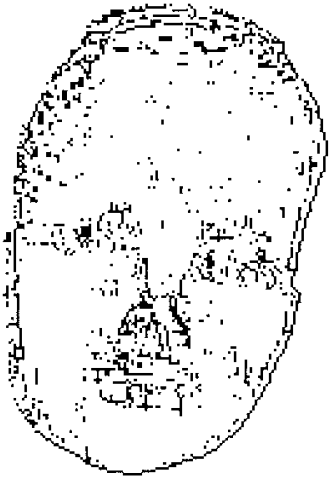

(g) (d)

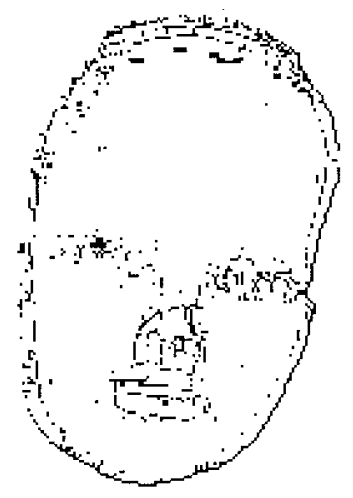

(h)

Fig. 8. Adaptive filtering result of a model head object using the PUF procedure. (a) Range image captured by a laser-stereo range finder. (b) Filtering result of applying the 1-D PUF opening procedure to each row of 8(a). (c) Filtering result of applying the 1-D PUF opening procedure to each column of $8(\mathrm{~b})$. (d) Filtering result using the procedure of 1-D PUF-closings to filter the rows and columns of 8(c). (e)-(h) Corresponding zero-crossings of the second-order derivative of $8(a)-(d)$, respectively.

many signal processing applications, for example, in the digital image compression area [11], [26]. In addition, notice that one of the reasons why the 1-D row-column decomposition is popular in signal processing is that its time complexity is smaller than using 2-D operations.

\section{EXPERIMENTAL RESULTS}

In this section, we describe how the PUF procedure was applied to the area of adaptive signal filtering. Two types of signals were presented in our experiments. First, the PUF procedure was applied for adaptive filtering of noisy range images. Second, it was applied to adaptive filtering of an intensity image corrupted with pepper and salt noises.

\section{A. Adaptive-Smoothing of Noisy Range Images}

In this experiment, the range images were obtained from a laser-stereo range finder [4]. Due to the limited precision of such a 3-D scanning system, some bumping noises could occur in the range of about $1.0 \mathrm{~mm}$ if the distance between the camera and the object was about $1.0 \mathrm{~m}$. To show the object surface in a clear way, a standard shading technique known as the Phong shading [9] was used to shade the objects contained in range images. The SE's used in this experiment for adaptive signal filtering were also circular ones, and the procedure used was PUF close-open. The criterion used for feature detection was the volume of a connected highly varying portion as defined in (2.6), and the threshold was fixed to be $T_{i ; m}=5.0 \mathrm{~mm}^{2}$. The largest radius used in this experiment was $\boldsymbol{r}=20 \mathrm{~mm}$, and the SE size-reduction factors used were $k_{a}=0.5$ and $k_{i}=1$. In Fig. 8(a), a noisy range image of a model head is shown, where the height of this model head was about $200 \mathrm{~mm}$. After the filtering of each row of Fig 8(a) using the procedure of PUF opening, the result is shown in Fig. 8(b). Fig. 8(c) shows the result obtained by filtering each column of Fig. 8(b) using the PUF opening. It can be observed that the noises have been greatly removed, as shown in Fig. 8(c). Finally, Fig. 8(d) shows the filtering result after applying the PUF close-open procedure [i.e., using PUF closing to filter the output signal shown in Fig. 8(c)]. In principle, the bumping noises that occurred in a range image could be gradually removed through this procedure. To make sure that the resulting object surface was smoother than the original one, the zero crossings of the second-order derivatives of Fig. 8(a)-(d) are shown in Fig. 8(e)-(h), respectively. The set of these zero crossings is the union of the sets of the 1-D zero-crossings computed through each row and each column, where a point $x$ is a zero crossing if $f^{\prime \prime}(x+1)>0$ and $f^{\prime \prime}(x-1)<9$, and $f^{\prime \prime}(x+1)-f^{\prime \prime}(x-1)>e$ (where $f^{\prime \prime}$ is the second derivative of $f$, and $e$ is a threshold). From Fig. 8(e)-(h), we can observe that the original range image 


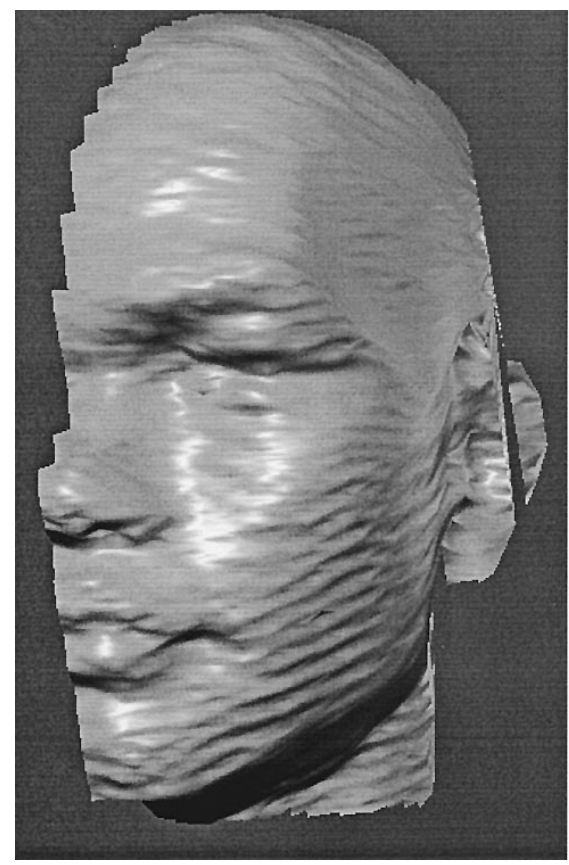

(a)

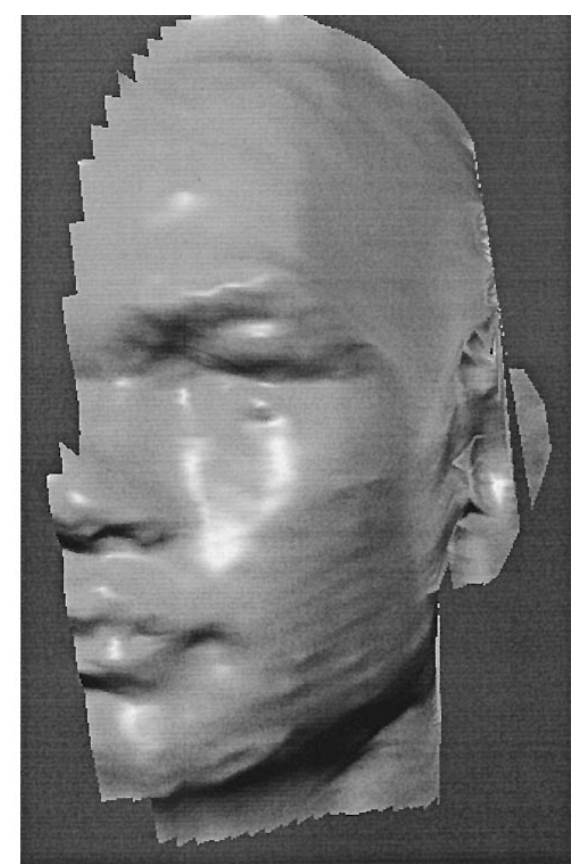

(b)

Fig. 9. (a) Original range image. (b) Smoothed range image using the PUF close-open procedure.

has been smoothed progressively. Fig. 9 shows the filtering result of a range image containing another model head object.

\section{B. Adaptive Noise-Removal of Intensity Images}

Fig. 10(a) is an intensity image corrupted by $5 \%$ pepper and salt noises, where the pepper noises have gray level 0 , and the salt noises have gray level 255. Flat SE's were used in this experiment. Notice that if the size of a flat SE was $w$, then the opening could remove a connected salt noise with size less than $w$. The idea of $\mathrm{SE}$ assignment in this experiment uses a flat $\mathrm{SE}$ with size $w$ to remove noises with size $w-1$. To achieve this goal, the PUF procedure was slightly modified such that the size of the SE was decreased by one in each iteration, i.e., $s_{i+1}(x)=s_{i}(x)-1$, in this experiment. In this experiment, the operation of $\mathrm{V}$ open-close was used. The size of the largest SE used was 3 in MV closing and was 5 in MV opening since the precedence MV closing could enlarge the size of the pepper noises. The feature-detection criterion of the PUF opening (or PUF closing) procedure used here is that a grain $C$ is a noise if there are $w-1$ salt (or pepper) noises in $C$, where $w$ is the current SE size; otherwise, $C$ is a feature and should be preserved for processing in the next iteration. Fig. 10(b) shows the filtering result after applying the 1-D PUF opening procedure for each row and column of Fig. 10(a). Notice that the pepper noises are well removed in Fig 10(b). Finally, Fig. 10(c) shows the filtering result after PUF open-close, and it is clear that the pepper and salt noises have all been removed.

\section{CONCLUSIONS AND Discussion}

In this paper, we introduced a well-organized approach for adaptive signal and image filtering using vertically invariant morphological filters. Adaptive smoothing is a nonlinear fil- tering scheme that can achieve feature-preserving smoothing. In principle, MV openings and MV closings have intuitive geometric interpretations and can provide different filtering scales with respect to different spatial positions. Hence, they are suitable for adaptive signal filtering. Basically, SE assignment is the key issue in applying MV openings or MV closings to adaptive signal smoothing. To solve the SE-assignment problem, we propose the PUF procedure in this paper. The PUF procedure can gradually fill the umbra of a signal with a set of overlapping SE's that are larger to smaller in scale. We have presented several examples and have shown that the PUF procedure can successfully reduce the bumping noises without oversmoothing the signal.

In fact, the PUF procedure also equivalently performs a multiscale feature-extraction scheme. This is because the PUF procedure is a process that reconstructs the umbra of a signal in a coarse to fine manner. Basically, the PUF procedure can be used to extract features at different scales by applying the following strategy:

The features extracted in prior iterations of the PUF procedure are referred to as the features of larger scales, and the features extracted in the later iterations are referred to as the features of smaller scales.

In principle, the types of features extracted in the PUF procedure are the highly varying parts (or the roof edges [16]) of a signal. An important characteristic of the features extracted by a PUF procedure is that no additional features will be introduced when the scales varying from larger to smaller. That is, all the smaller scale features are contained in the larger scales features. Hence, it obeys the multiscale property in the scale space. In fact, although many filtering approaches have focused on the multiscale property, the discussion of this scheme was usually restricted to the features caused by 


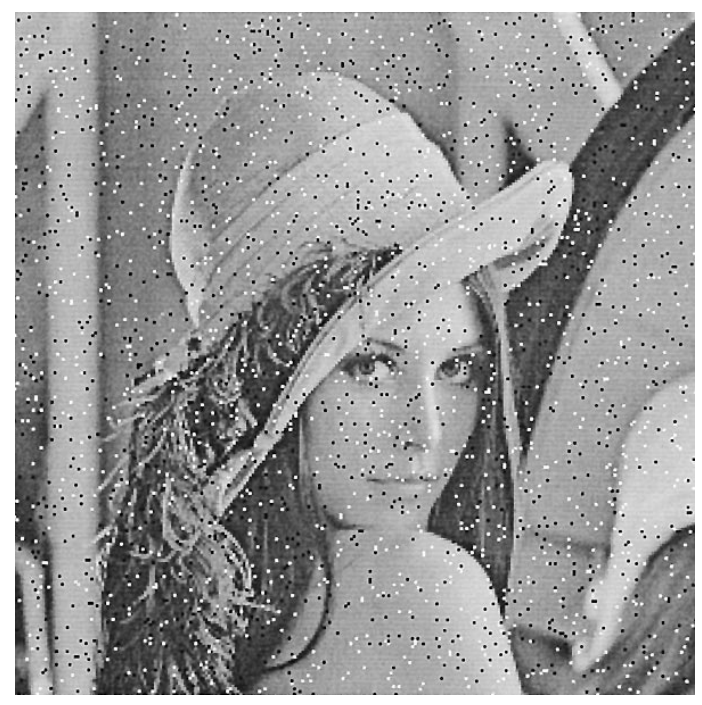

(a)

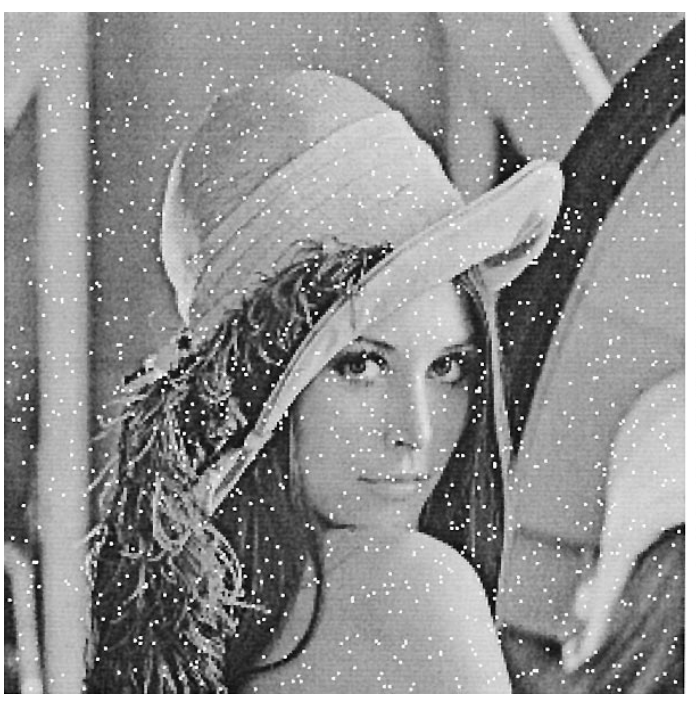

(b)

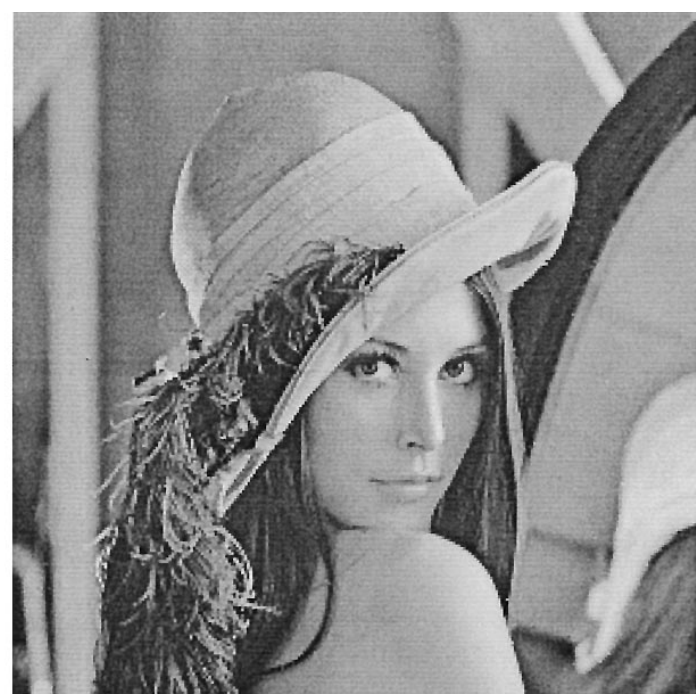

(c)

Fig. 10. Noise removal of an intensity image corrupted by pepper and salt noise using the PUF procedure.

discontinuities (e.g., the step edges) of a signal. However, by using the PUF procedure, the multiscale property can be achieved for the roof edges. Basically, this property does not create artificial features during the filtering process. Hence, the PUF procedure is a good tool to extract roof-edge features in a multiscale way and is also suitable for removing roof-edge-like noises such as the bumping noises occurring in different scales. The PUF procedure is a fast adaptive smoothing approach. Since, in general, only a few iterations are needed to achieve the filtering purpose in the PUF procedure, and because the sizes of the domains of the signals decayed very fast through the iterative process, the PUF procedure can be implemented in low-order time.

To sum up, we have introduced a hierarchical umbrareconstruction scheme that can be successfully implemented through mathematical morphology and can be appropriately applied to adaptive signal smoothing. The proposed approach is fast, easy to implement, and can remove noises while preserving important features. In addition, it can also be used for multiscale feature extraction. The PUF procedure proposed in this paper is a general multiscale signal representation tool, and hence, it has great potential for not only adaptive signal smoothing but many other signal processing applications as well.

\section{REFERENCES}

[1] P. J. Besl, Surfaces in Range Image Understanding. New York: Springer-Verlag, 1988, sec. 4.1.

[2] C. S. Chen, Y. P. Hung, and J. L. Wu, "Space-varying mathematical morphology for adaptive smoothing of 3D range data," in Proc. First Asia Conf. Comput. Vision, Osaka, Japan, 1993, pp. 39-42.

[3] _ _ "Extraction of corner-edge-surface structure from range images using mathematical morphology," IEICE Trans. Inform. Syst., Special Issue on Machine Vision Applications, vol. E78-D, pp. 1636-1641 1995.

[4] _ "A 3D range data acquisition system combining laser lighting and stereo vision," J. Chin. Inst. Elect. Eng., vol. 3, no. 2, p. 157-168, 1996.

[5] C. S. Chen, J. L. Wu, and Y. P. Hung, "Theoretical aspects of the gray-level morphological $\mathrm{V}$-opening and its applications on adaptive 
signal filtering," in Proc. 1995 IEEE Workshop Nonlinear Signal Image Process., Noes Marmaras, Halkidiki, Greece, 1995, pp. 42-45.

[6] _ "Statistical analysis of space-varying morphological openings with flat structuring elements," IEEE Trans. Signal Processing, vol. 44 pp. 1010-1014, Apr. 1996

[7] M. H. Chen and P. F. Yan, "A multiscaling approach based on morphological filtering," IEEE Trans. Pattern Anal. Machine Intell., vol. 11, pp. 694-700, 1989

[8] E. J. Coyle, J. H. Lin, and M. Gabbouj, "Optimal stack filtering and the estimation and structural approaches to image processing," IEEE Trans. Acoust., Speech, Signal Processing, vol. 37, pp. 2037-2066, 1989.

[9] J. D. Foley, A. V. Dam, S. K. Feiner, and J. F. Hughes, Computer Graphics, Principles and Practice, 2nd. ed. Reading, MA: AddisonWesley, 1990.

[10] C. R. Giardina and E. R. Dougherty, Morphological Methods in Image and Signal Processing. Englewood Cliffs, NJ: Prentice-Hall, 1988.

[11] R. C. Gonzales and R. E. Woods, Digital Image Processing. Reading, MA: Addison-Wesley, 1992.

[12] W. E. L. Grimson and T. Pavlidis, "Discontinuous detection for visual surface reconstruction," Comput. Vis., Graph., Image Process., vol. 30 , pp. 316-330, 1985

[13] R. M. Haralick and L. G. Shapiro, Computer and Robot Vision, vol. 1. Reading, MA: Addison-Wesley, 1992, ch. 5.

[14] H. J. A. M. Heijmans, "Connected morphological operations for binary images," from http://www.cwi.nl/ftp/morphology/report/, 1997.

[15] H. J. A. M. Heijmans and C. Ronse, "The algebraic basis of mathematical morphology, I. Dilations and erosions," Comput. Vis., Graph., Image Process., vol. 50, pp. 245-295, 1990.

[16] R. Krishnapuram and S. Gupta, "Morphological methods for detection and classification of edges in range images," J. Math. Imag. Vision, vol 2, pp. 351-375, 1992.

[17] S. Z. Li, "On discontinuity-adaptive smoothness priors in computer vision," IEEE Trans. Pattern Anal. Machine Intell., vol. 17, pp. 576-586, 1995.

[18] P. Maragos, "A representation theory for morphological image and signal processing," IEEE Trans. Pattern Anal. Machine Intell., vol. 11, pp. 586-599, 1989.

[19] _ "Pattern spectrum and multiscale shape representation," IEEE Trans. Pattern Anal. Machine Intell., vol. 11, pp. 701-716, 1989.

[20] G. A. Mastin, "Adaptive filters for digital image noise smoothing: An evaluation," Comput. Vis., Graph., Image Process., vol. 31, pp. 103-120, 1985.

[21] A. Morales and R. Acharya, "Non-linear multiscale filtering using mathematical morphology," in Proc. 1992 IEEE Comput. Soc. Conf. Comput. Vision Pattern Recogn., CVPR, Champaign, IL, 1992, pp. 572-578.

[22] _ "Statistical analysis of morphological openings," IEEE Trans. Signal Processing, vol. 41, pp. 3052-3056, 1993.

[23] M. Niedzwiecki and W. A. Sethares, "Smoothing of discontinuous signals: The competitive approach," IEEE Trans. Signal Processing, vol 43, pp. 1-13, Jan. 1995.

[24] R. A. Peters, II, "A new algorithm for image noise reduction using mathematical morphology," IEEE Trans. Image Processing, vol. 4, pp. 554-568, Apr. 1995

[25] S. M. Philippe, J. S. Chen, and G. Medioni, "Adaptive smoothing: A general tool for early vision," IEEE Trans. Pattern Anal. Machine Intell., vol. 13, pp. 514-529, 1991 .

[26] M. Rabbani and P. W. Jones, Digital Image Compression Techniques. Bellingnam, WA: SPIE, 1991

[27] A. Restrepo and A. C. Bovik, "Adaptive trimmed mean filters for image restoration," IEEE Trans. Acoust., Speech, Signal Processing, vol. 36, pp. 1326-1337, 1988.

[28] C. Ronse and H. J. A. M. Heijmans, "The algebraic basis of mathematical morphology, II. Openings and closings," CVGIP: Image Understanding, vol. 54, pp. 74-97, 1991.

[29] J. Serra, Ed., Image Analysis and Mathematical Morphology, Vol. 2, Theoretical Advances. New York: Academic, 1988.

[30] J. Song and E. J. Delp, "The analysis of morphological filters with multiple structuring elements," Comput. Vis., Graph., Image Process., vol. 50, pp. 308-328, 1990

[31] D. C. C. Wang, A. H. Vagnucci, and C. C. Li, "Gradient inverse weighted smoothing scheme and the evaluation of its performance," Comput. Vis., Graph., Image Process., vol. 15, pp. 167-181, 1981.

[32] D. Wang, V. Haese-Coat, A. Bruno, and J. Ronsin, "Texture classification and segmentation based on iterative morphological decomposition," J. Visual Commun. Image Represent., vol. 4, pp. 197-214, 1993.

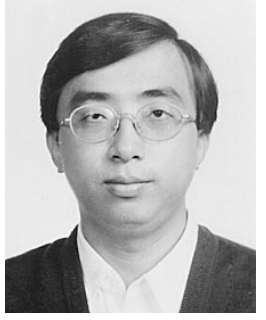

Chu-Song Chen (M'96) received the B.S. degre in control engineering from National Chiao-Tung University, Hsinchu, Taiwan, R.O.C., in 1989. He received the M.S. degree in 1991 and the Ph.D. degree in 1996, respectively, both from the Department of Computer Science and Information Engineering, National Taiwan University, Taipei.

$\mathrm{He}$ is now a postdoctoral fellow with the Institute of Information Science, Academia Sinica, Nanking, Taipei. His research interests include multimedia signal processing, computer vision, and virtual reality.

Dr. Chen received the good paper award and the outstanding paper award of the Image Processing and Pattern Recognition (IPPR) Society of Taiwan in 1995 and 1997, respectively. He received the best paper award of the Image Processing and Application Association (IPAA) of Taiwan in 1997.

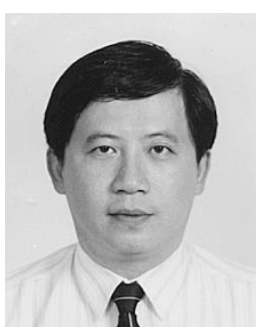

Ja-Ling Wu (SM'96) received the B.S. degree in electronic engineering from Tamkang University, Tamshoei, Taiwan, R.O.C., in 1979, and the M.S and $\mathrm{Ph} . \mathrm{D}$. degrees in electrical engineering from Tatung Institute of Technology, Taipei, Taiwan, in 1981 and 1986, respectively.

From 1986 to 1987, he was an Associate Professor of the Electrical Engineering Department, Tatung Institute of Technology. Since 1987, he has been with the Departmnt of Computer Science and Information Engineering, National Taiwan University, Taipei, where he is presently a Professor and the Director of the Communications and Multimedia Laboratory. From 1996 to 1998, he was the Head of the Department of Information Engineering, Nation Chi Nan University, Puli, Taiwan. He has published more than 200 technique and conference papers. His research interests include neural networks, VLSI signal processing, image coding, data compression, and multimedia systems.

Prof. Wu was the recipient of the Outstanding Young Medal of the Republic of China and the Outstanding Research Award sponsored by the National Science Council in 1987 and 1994, respectively. He was the recipient of the Award for Distinguished Information People in 1993, the Special Long-Term Award for Collaboratory Research in 1994, the Best Long-Term paper Award in 1995, and the Long-Term Medal for Distinguished Researchers in 1996, all sponsored by the Acer Corporation.

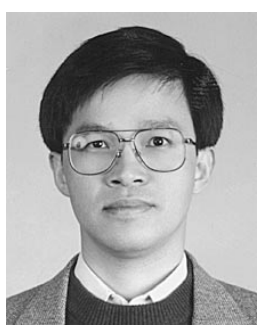

Yi-Ping Hung (M'89) received the B.S. degree in electrical engineering from National Taiwan University, Taipei, R.O.C., in 1982. He received the M.S. degree from the Division of Engineering, the M.S. degree from the Division of Applied Mathematics, and the Ph.D. degree from the Division of Engineering, Brown University, Providence, RI, in 1987, 1988, and 1989, respectively.

$\mathrm{He}$ then joined the Institute of Information Science, Academia Sinica, Taipei, Taiwan, and became a Research Fellow in 1997. He served as the Deputy Director of the Institute of Information Science from 1996 to $1997 . \mathrm{He}$ has been teaching in the Department of Computer Science and Information Engineering at National Taiwan University since 1990 and is now an Adjunct Professor. He has published more than 70 technical papers in the fields of computer vision, pattern recognition, image processing, and robotics. In addition to the above topics, his current research interests also include visual surveillance, virtual reality, human-computer interface, and visual communication.

Dr. Hung received the Outstanding Young Investigator Award given by Academa Sinica in 1997. 